\title{
RECURRENT MULTIFOCAL OSTEOMYELITIS AND TAKAYASU'S ARTERITIS: CASE REPORT
}

William Barros Hyppolito Ferreira ${ }^{1, \star}$, André Marun Lyrio${ }^{1}$, Vanessa Félix Nascimento Coelho ${ }^{1}$, Maria Verônica Russo Macchi Carolina Pellisson Carvalho ${ }^{1}$, Nayara Mota Carvalho ${ }^{1}$, Rubens Bonfliglioli ${ }^{1}$

1.Pontifícia Universidade Católica de Campinas, Campinas (SP), Brazil.

*Corresponding author:wf_ferreira@hotmail.com

\section{BACKGROUND}

Recurrent multifocal osteomyelitis is a rare autoinflammatory bone disorder that mainly affects children, but it can occur in all age groups to a lesser extent. The clinical spectrum ranges from asymptomatic bone lesions to more severe forms of recurrent multifocal osteomyelitis. It is believed that the interleukin 10 and interleukin 1 pathways may be involved in the etiology as part of systemic inflammation.

\section{CASE REPORT}

A 31-year-old female patient, previously without comorbidities, was admitted to hospital in June 2018 due to arthritis in the left sternoclavicular joint, with osteomyelitis and abscess invading the anterior mediastinum and left sternocleidomastoid muscle. During the investigation of the condition, a magnetic resonance angiography of the aorta and cervical vessels was performed, which showed extensive vasculitis of large vessels, with aortic stenosis, renal arteries, celiac trunk, left and right subclavian arteries, common carotids, suggestive of Takayasu, without clinical symptoms. She underwent treatment with antibiotic therapy with ceftriaxone and teicoplanin for 6 weeks, in addition to starting prednisone $60 \mathrm{mg} /$ day, but in the outpatient follow-up all cultures were sterile. On an outpatient return in September 2018, she was asymptomatic, azathioprine was introduced and a gradual reduction in the dose of prednisone was started until it was withdrawn in May 2019. During the period, maintenance of azathioprine was chosen instead of methotrexate, since the patient did not wish to use an effective contraceptive method. In October 2019, she presented a new manifestation of pyogenic arthritis in the right sternoclavicular joint, with osteomyelitis and abscess in the right clavicle, cultures all negative, but due to gravity, antibiotic therapy was performed with ciprofloxacin and teicoplanin for 6 weeks, in addition to suspending azathioprine and reintroducing prednisone $40 \mathrm{mg} /$ day during hospitalization. On an outpatient return visit, the introduction of methotrexate was chosen and the diagnostic hypothesis of recurrent multifocal osteomyelitis was raised, with zoledronic acid being administered in a single dose. In July 2020, methotrexate was suspended due to pregnancy, and the dose of prednisone was increased to $20 \mathrm{mg} / \mathrm{day}$, which was maintained until the end of pregnancy. In March 2021, she had a cesarean delivery, uneventful, currently asymptomatic and undergoing corticosteroid dose reduction, scheduled to perform a new control angiography that was postponed due to pregnancy.

\section{CONCLUSION}

Recurrent multifocal osteomyelitis should be considered in the context of recurrent bone infections, without identifying an etiologic agent. This report not only highlights a rare example of recurrent multifocal osteomyelitis in adults, but also presents its association with Takayasu's arteritis.

\section{KEYWORDS}

Recurrent multifocal osteomyelitis, Takayasu's arteritis, Vasculitis. 